\title{
Erratum to: Surgical approach is superior to palliative treatment in oligometastatic lung cancer
}

Till Ploenes · Alberto Lopez-Pastorini · Erich Stoelben

Published online: 10 November 2016

(C) Springer-Verlag Wien 2016

\section{Erratum to: \\ DOI 10.1007/s10353-016-0437-X}

In the version originally published online, the legends to the two parts of Fig. 1 did not match the figures. Part a shows the FEV1 in liters and part $b$ the FEV1 in per cent. Moreover, the legend to Fig. 2 belongs to Fig. 4 and vice versa. The article has been updated in the meantime.

Conflict of interest The author(s) declare that they have no competing interests.

The online version of the original article can be found under http://dx.doi.org/10.1007/s10353-016-0437-x.

T. Ploenes, MD $(\bowtie) \cdot$ A. Lopez-Pastorini $\cdot$ E. Stoelben Lungclinic Merheim, Department of Thoracic Surgery, Lung Clinic Cologne, Kliniken der Stadt Köln gGmbH, Cologne Merheim Hospital, Faculty of Health/School of Medicine, Witten/Herdecke, Campus Cologne, Ostmerheimerstraße 200, 51109 Köln, Germany Till_ploenes@gmx.de 